\title{
Syntactic Interpolation of Fractal Sequences
}

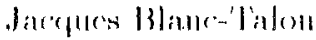 \\ GT $\mathrm{G} / \mathrm{GIP}$ \\ 16 bis, Avenue Prieur cle la côte d'or, \\ 9414, Arcueil, FRANCE \\ blancoetca.fr
}

\begin{abstract}
A$ grammatical inference algorithm is dewerbed which computes a context-free grammar interpolating fractal oncolings. The "Tho" tality" of the initial curve is captured by the symbolic part of the algoribm while the approximation is performod by the mumeteal part. Hybridization of the algorithm consists in cxchanging some information between the two parts, i.c. hy translating the eigcnvalue sariations of the growth matrix into syntactic variations while checking the compatibility with the symbolic system already inferred.
\end{abstract}

\section{Introduction}

If a lot of works have becn published on patien recognition in the previous decades, there are few available on the recognition of fractal sets. Fractals are easy to generate and yield nice art displays but once the wonder has vanished the fascinating inverse problem of computing the models from real data remains, which is still unviolated. Even with models as simple as IFSs [12] the inverse problem may lead to untractable computations; it is thus impossible to compute more advanced models, even if desirable for the sake of generalization and for practical reasons. Nevertheless, the key to unlock fractal models seems to be the relationship between their internal structure and the basic elements they are made of.

Structural Pattern Recognition (SPR) gathers a wide set of techniques whose distinctive feature is to give major importance to some special information against the set of patterns to process. In fact, recognition is achieved by combining information of two difrcrent nalures: firsty some disparale inlormation consisting in a decomposition over a set of primitive paltems and called the alphabet and secondly some long-range information describing relationships between subsets of patterns called the structure. Accepted patterns must present both alphabetical and structural information compatible with the model but most of the time, the two are straightforwardly juxtaposed since they do not belong to the same physical or mathematical space.

In this paper, a method for computing models from "fractal encodings" of sets is cliscussed. (Hiven a sequence of encodings, the underlying structure of the 
model is computed first, then angmented with disparate information. In order to make the first and the second one compatible, we use a syntactic model to provide a unified framework for dealing with such information at various

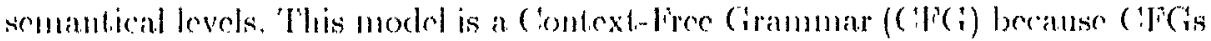
are appropriate to fractal modelling' (they allow intricate recursive derivations) and because any "mon fractal" noise may be modelled by a regular language (which dors not change the overall structure [i]). 'The syntactic optimization runs together with a numerical algorithm so as to infer the granmatical model.

'This method has been tested on encodings of $2 \mathrm{D}$ curves generated by justtouching IFSs. However, the practical application which gave birth to this study was far more complex: it dealt with the modeling of gold ore distribution in a mining area [11]. Because such a distribution exhibits fractal features, syntactic tochniques seemed suitable for modeling huge data sets.

'The paper is organized as follows. 'Theortical backgromel is recalled in Sect. 2. An encoding process which extracts syntactic and structural information simultanconsly is discussed in Sed. 3. Sections 4 and 5 detail the very rore of the inference method. In the conchusion, we cmphasize the nse of syntactic models for processing fractal informalion.

\section{Preliminaries}

L-systems are rewriting machines which can be used for generating self-affine fractal curves $[5,8]$. For instance, Fig. 1 shows the attractor of a D0L-system whose rules once concatenated yield the sentence "SSPR should take place in Australia", the initial word being "Sydney". DOJ,s [14] are among the simplest Lsystems. Although "innocent-looking", they allow the computation of sequences with rather complex combinatorial properties. Moreover, the diversity of curves gets wider if several morphisms are used.

In this paper, $T$ is a finite set and $T^{\star}$ the free monoid of words; 1 is the null letter. For $t \in T, x \in T^{*},|x|$ denotes the length of $x$ and $|x|_{t}$ the number of occurrences of $t$ in $x$. Also, $x[k]$ is the subword made of the $k$ first letters of x. A D0L is a triple $(T, h, s)$ which generates the language $\mathcal{L}(D)=\left\{h^{n}(s)=\right.$ $\left.h \circ h^{n-1}(s), n \geq 0\right\}$.

$d_{L}(x, y)$ clenotes the Levenstein clistance (sometimes called edition distance) between strings $x, y \in T^{*}$. This distance is defined very intuitively by mons of three basic operations, del (-etion), ins (-ertion) and subs (-titution):

$$
\begin{aligned}
& \operatorname{del}(1)=\{1\} \\
& \forall a \in T, \operatorname{del}(a)=\{1\} \\
& \forall u, v \in T^{*} \backslash\{1\}, \operatorname{del}(u v)=\operatorname{del}(u) v+u \operatorname{del}(v) \\
& \operatorname{ins}(1)=T \\
& \forall a \in T, \operatorname{ins}(a)=T a+a T \\
& \forall u, v \in T^{*} \backslash\{1\}, \operatorname{ins}(u v)=\operatorname{ins}(u) v+u \operatorname{ins}(v)
\end{aligned}
$$

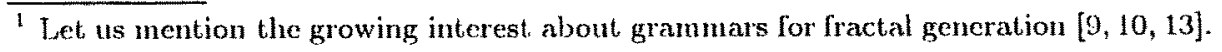




$$
\begin{aligned}
& \operatorname{subs}(1)=\{1\} \\
& \forall a \in T, \operatorname{subs}(a)=T \backslash\{a\} \\
& \forall u, v \in T^{*} \backslash\{1\}, \text { subs }(u n)=\operatorname{sub} s(u) n+u \text { sulss }(u)
\end{aligned}
$$

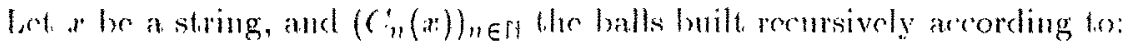

$$
\begin{aligned}
C_{i n}(x) & =\{x\} \\
G_{n, n \geq 1}(x) & =\operatorname{dd}\left(C_{n-1}(x)\right)+\operatorname{ins}\left(C_{n-1}(x)\right)+\operatorname{subs}\left(C_{n-1}(x)\right)
\end{aligned}
$$

'Then the Levenstem distance between $x$ and $y$ is:

$$
d_{1}(x, y)=\inf \left\{n \in\left[y \mid y \in C_{n}(x)\right\}\right.
$$

Let us recall a few results about the perturbation of the roots of a polynomial and the eigenvalues of a matrix (sec [15] for instance). In the following, vectors are in bold font; the contries of any matrix $A$ lic in brackets: $\left(a_{i, j}\right)$, its transpose is $A^{T},|A|$ is the matrix whose clements are $\left|a_{i, j}\right| . l_{n}$ is the identity matrix of size $n \times n, \sigma(A)$ is the spectrmm of $A . \delta_{i, j}$ is the Kronedker delta.

II. A clenotes either the usual Enclidean vector norm or the Frobonius matrix norm; the latier has been chosen since it satisfies the triangle inecuality for matrix multiplication.

We shall use the following proposition and theorems:

Proposition 1. Let $A \in \mathcal{M}_{n}(\mathbb{R}), \mathbf{b}, \mathbf{c} \in \mathbb{R}^{n}$ then (formally) :

$$
\left(A+\mathrm{cb}^{T}\right)^{-1}=A^{-1}\left(I_{n}-\frac{\mathrm{cb}^{T} A^{-1}}{1+\mathrm{b}^{T} A^{-1} \mathrm{c}}\right)
$$

Theorem 1 (Banor-Skeel). Let $A$ be nonsingular; let $A \mathbf{x}=1$ and $(A+E) \tilde{\mathbf{x}}=$ $\mathrm{b}+\mathrm{e}$. If for some nonnegative $S, \mathrm{~s}$ and $\epsilon:|\mathrm{H}|<\epsilon S^{\prime}$ and $|\mathrm{e}|<\mathrm{cs}$ and in addition . $\left\|\left|A^{-1}\right| s^{\prime}\right\|<1$ then

$$
\|\tilde{\mathrm{x}}-\mathrm{x}\| \leq \frac{c\left\|\left|A^{-1}\right|(S|\mathrm{x}|+\mathbf{s})\right\|}{1-\epsilon\left\|\left|\Lambda^{-1}\right| S\right\|}
$$

Many theorems yield inequalities for the roots of perturbed polynomials; the following recent one refines Ostrowski's. Let the Bombieri's norm of a polynomial $P=\sum_{i=1}^{n} a_{i} x^{i}$ be:

$$
[P]_{B}=\sqrt{\sum_{i=0}^{n} \frac{i !(n-i) !}{n !}\left|a_{i}\right|^{2}}
$$

Theorem 2 (Beauzamy [4]). Let $P$ and $Q$ be two polynomials defined as: $P(x)=\prod_{i=1}^{n}\left(x-x_{i}\right)$ and $Q(x)=\prod_{i=1}^{n}\left(x-y_{i}\right)$ and such that $[P-Q]_{B}<\epsilon$. If all the roots of $P$ are distinct and if

$$
\epsilon \leq \frac{1}{2 n} \min _{j} \frac{\left|P^{\prime}\left(x_{j}\right)\right|}{\left(1+\left|x_{j}\right|^{2}\right)^{(n-1) / 2}}
$$

then for every $j$ a root $y_{i}$ of $Q$ is such that

$$
\left|x_{j}-y_{i}\right|<2 n \frac{\left(1+\left|x_{j}\right|^{2}\right)^{n / 2}}{\left|P^{\prime}\left(x_{j}\right)\right|} \epsilon
$$




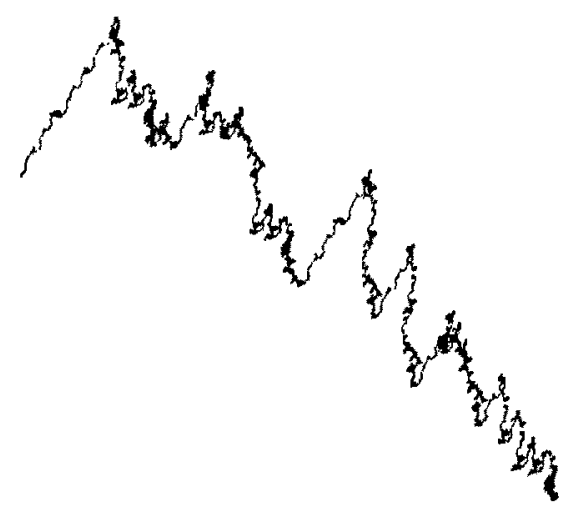

Figure1. Iterating the rules "SSPIR should take place in Australia" on axion "Sychey" $(41,357$ symbols $)$

\section{Encodings of Real Sets}

The general idea of fractal coding is given in [3]. We have introduced in previous papers $[5,7]$ an image encoding method based on a recursive segmentation of 2D sets. This method yields non-balanced quadtrees [1] whose terminal leaves are subimages taken within the original image. Quadtree encoding is easy to implement, quadtrees meet accuracy issues since the general quadtree is but the image itself and mapping any quadtree to a screen can be performed very efficiently. In the present case, the whole image is assumed to be a (possibly infinitc) collection of self-alfine copies of a few source subimages.

The comparison between images is performed by an enhanced correlation operator which is able to manage rotation and scaling of the sets to be compared. The leaves are the terminal letters of the final grammar while the branching nodes of the quadtree are considered as non-terminal symbols. They are rewritten as a word of the nodes (terminal or not) they are giving access to. Figure 2 shows a self-affine curve; rectangular boxes are terminal leaves linked by correlation maximization.

This method has given satisfactory results for self-affine sets but unfortunately it cannot be applied to multifractal sets which are anything but self-affine! Thus, the algorithm discussed below makes no use of any extra structural information delivered with the encodings. Instead, it only makes the assumption that the encodings reflect the (multi-) "fractality" of some initial phenomenon one wants to model. In the formal languages terminology, "fractality" implies "iteration" and requires at least the use of context-free grammars [7].

Nevertheless, it is always possible to encode a Jordan curve as a sequence of words highlighting some iteration process (provided there is some theoretical justification in doing so !) as pointed out in [6]. For instance, the number of symbols $N(\epsilon)$ for encoding a fractal curve as a function of a small line segment behaves as $A \epsilon^{-D}$ where $D$ is the fractal dimension; various algorithms are available for computing $D$ and for performing Freeman encoding. 
As the curve is assumed to be interpolated by a non-erasing Dol-system, Perrou-Frobenius' theorem yields $N(\epsilon) \sim B\left(\lambda_{\max }\right)^{k}$ for some $k, \lambda_{\text {max }}$ being the (positive) greatest eigenvalue of the growth matrix (see Sect. 4). Sime $\epsilon$ is a parameter of the encoling algorithm ( $\epsilon$ may be a function of the encoding accuracy), it is possible to plot $(\log (c), \log (N(c)))$ so as to find $\lambda_{\text {max }}$ the best inleger sequence $k_{1}, k_{2}, \ldots$, and the relited encolings are computed actorlingly. We performed such a process succesfilly on just-tonching liS curves [5].

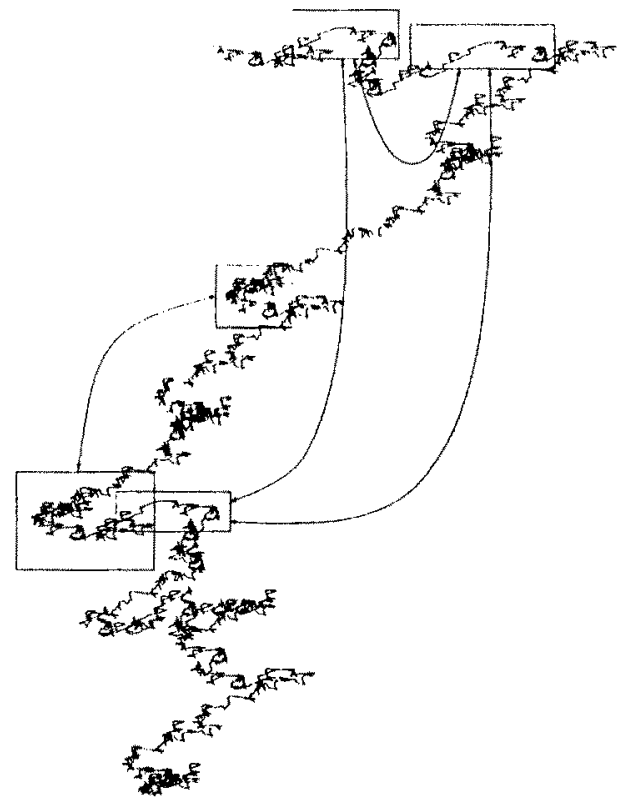

Figure2. Possible correlation locations in a self-affine curve

\section{DoL Computation and Grammatical Inference}

$\Lambda$ s an input, let us consider a sequence $S=\left\{x_{1}, x_{2}, \cdots, x_{p}\right\}$ of words over $T=\left\{t_{1}, t_{2}, \cdots, t_{n}\right\}$ (with $n+1<p$ ) and the related sequence of integer vectors: $\mathrm{g}_{k}=\left[\left|x_{k}\right|_{t_{1}},\left|x_{k}\right|_{t_{2}}, \cdots,\left|x_{k}\right|_{t_{n}}\right], \quad 1 \leq k \leq p . S$ can be mapped to $\mathbb{R}^{n}$ by using an expansive morphism $K: T^{\star} \rightarrow \mathbb{R}^{n \text { which }}$ is not needed to be detailed here. The "fractal space" is the complete space $\left(\mathbb{R}^{n}, h\right)$ where $h$ is the Ilausdorff metric. One assumes there is an approximation of $S$ by a regular D0L-sequence, i.e.

$$
x_{k}=\mu_{k} \circ \phi^{k}\left(\tilde{x}_{0}\right), 1 \leq k \leq p,
$$

with $\phi: T^{\star} \rightarrow T^{\star}$ a non-erasing morphism, $\tilde{x}_{0}$ some initial word ${ }^{2}$ and $\mu_{k}$ a transduction such that

${ }^{2}$ Please note that symbol " " means that the adressed value is the one related to the optimal case, i.e. without noise. 


$$
d_{L}\left(\mu_{k} \circ \phi^{k}\left(\tilde{x}_{0}\right), \phi^{k}\left(\tilde{x}_{0}\right)\right)<q, 1 \leq k \leq p .
$$

Previons equation can be rewritten in the numerical spare as

$$
\mathrm{g}_{k}=M^{k} \dot{\mathrm{g}}_{3}+O_{k}, 1 \leq k \leq 1
$$

In this equation $M \in \mathcal{M}_{n}(\Gamma), g_{k} \in[]^{n}, \tilde{g}_{11}$ is an initial vector and $\theta_{k}$ stands for some bounded noise $\left(\sup _{1 \leq k \leq r}\left|O_{k}\right|=q<\infty\right)$ whose distribution is assumed to be uniform over $T$. One must notice that this condition is rather general: the noise may be anything but a cascading process. In general, suj $j_{k}\left|0_{k}\right| \leq$ $\sup _{l} d_{L}\left(\mu_{l} \circ \phi^{l}\left(x_{0}\right), \phi^{l}\left(x_{0}\right)\right)$. It is important that $q$ is finite as stated by:

Proposition 2 (Blanc-Talon [7]). If $\left(u^{\prime \prime}\right)$ is an increasing scqucuce (acrovding 10 S) whose limit is $u$, and if $\left(v^{n}\right)$ is a sequence such that $\exists p, m \in \mathbb{N}, \forall n>$ $m, d_{L}\left(u^{n}, v^{n}\right) \leq p$, then their mappings $K\left(u^{n}\right)$ and $K\left(v^{n}\right)$ in fr: $^{n}$ converge to the sume "inve in the llatsdorff medrith.

$M$ is the growth matrix of morphism $\phi$ and its entrics are $\left|\phi\left(t_{i}\right)\right|_{i}$. Inferring the DoL-system in the perfect case $\left(\forall k, \theta_{k}=0\right.$ and $\left.q=0\right)$ can be achicved either in the symbolic space or in the numerical space. However, neither method works in case of noisy sequences. The new following approach consists in using the partial results computed by a numerical algorithm as the input of a symbolic method and vice-versa, as long as the result after the current pass is not rorrect. The maximum number of loops is $n(=\# T)$.

First, one delermines the characteristic polynomial $\tilde{P}$ of the perturbed natrix $\dot{M}$, written $R$ for sake of clarity, from the set of vectors $\mathrm{g}_{k}$. Since the noise has an upper limit, the method converges to the correct values: the coefficients of $\tilde{P}$ have continuous variations according to the noise, and so do the roots if this noise is small enough. Then, perturbed eigenvalues are computed, which are located in some Gerschgorin disks of the exact values we are looking for. We use a refined estimate of the disc for computing their variation range and possible integral matrices are constrained by this result. The symbolical part consists in determining the morphism rules with the greatest row sum; it is performed by considering the number of occurrences of subwords of increasing lengih.

Please notice that the proofs are given below in the scalar case which lightens the notations (a lot !). Instead of considering the sequence of vectors $\mathrm{g}_{k}$, one considers a sequence of vector entries $\left(g_{1}=\mathrm{g}_{1}(l), \cdots, g_{p}=\mathrm{g}_{p}(l)\right)$. This sequence also forms a vector; let $\mathrm{g}$ denote this vector. The following formulas are still valid for vectors, however in a more complex form. Given \#T, there is indeed a particular $l$ for which the whole method does work (i.e. matrix $G$ is nonsingular). Approximating $\tilde{P}$ by $P$ implies computing the best hyperplane through $g_{1}, \cdots, g_{p}$ :

$$
\frac{\partial}{\partial a_{m}} \sum_{k=n+1}^{p}\left(g_{k}-\sum_{l=1}^{n} a_{l} g_{k-l}\right)^{2}=0
$$


which yields:

$$
\mathrm{a}=C^{-1} \mathrm{n}=\left[(s q(i, j))_{i, j=1}^{n}\right]^{-1}(s q(i, 0))_{i=1}^{n}
$$

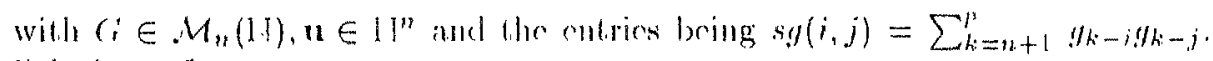
Solutions of

$$
x^{n}-\mathbf{a}^{T}\left(x^{k}\right)_{k=1}^{n-1}=x^{n}-\sum_{k=0}^{n-1} a k_{k} x^{n-k}=0
$$

are the estimated eigenvalues. One has $i=\left(i-\left(0 \theta^{T}+\mathrm{g} \theta^{T}+\theta \mathrm{g} T\right)\right.$. Perturbation of $\left(i^{-1}\right.$ can thus be computed by setting $A=G-\left(0 \theta^{T}+\mathrm{g} \theta^{T}\right), b=\mathrm{g}, c=-\theta \mathrm{in}$ (1) which allows Theorem 1 to yield the max of $\|\mathrm{a}-\tilde{a}\|$, that is the perturbation over polynomial coefficients. The next step) is to quantify the spectrum variation: assuming that the roots of $P$ are distinct 'Theorem 2 applies :

$$
\left|\lambda_{i}-\dot{\lambda}_{j}\right| \leq 2 n\left|\lambda_{i}\right| \frac{\left(1+\left|\lambda_{i}\right|^{2}\right)^{(n / 2)}}{\mid \sum_{k=1}^{n} k\left(k_{k} \lambda_{i}^{n-k} \mid\right.}|| n-i n|| .
$$

This result states that one can deduce the range of varialions of $\sigma(R)$ with respect to $\sigma(M)$ from the bounds of $\theta_{k}$. How is it possible to relate $\|R-M\|$ itself to the former? Since $\tilde{P}(\lambda)=\operatorname{Det}\left(M-I_{n} \lambda\right)$, taking derivates with respect to any $\tilde{a}_{m}$ on both sides yields:

$$
\begin{gathered}
\lambda^{m}+m \tilde{a}_{m} \lambda^{m-1} \frac{\partial \lambda}{\partial \tilde{a}_{m}}=\sum_{k=1}^{n} \operatorname{Det}(Q(k)), \\
\text { with } Q(k)=\left\{\begin{array}{ll}
m_{i, j}-\delta_{i, j} \lambda & i \neq k \\
\frac{\partial m_{i, j}}{\partial \tilde{a}_{m}}-\delta_{i, j} \frac{\partial \lambda}{\partial \tilde{a}_{m}} & i=k
\end{array} .\right.
\end{gathered}
$$

This expansion in terns of determinants of $Q(k)$ is very important since every line of $R$ reflects the related rule $\phi\left(t_{k}\right)$. Taking $\tilde{\lambda}$ in the neighborhood of every $\lambda_{i}$ allows us to determine the upper bound of the entries of $R$ as a function of the noise; the lower bound is always zero since $\phi$ is assumed to be non-erasing. Since $M \in \mathcal{M}(\mathrm{N})$, the problem can be solved by a minimization algorithm around initial entries of $R$. Simulated annealing has been used for finding $M$ which minimizes $\|\mathrm{g}(m+1)-M \mathrm{~g}(m)\|$ under the constraint $0 \leq$ $m_{i, j} \leq$ [variation given by (8)].

Thus, the whole algorithm can be summarized as follows.

1. Compute $\sigma(R)$ from $g(1), g(2), \cdots, g(p)$.

2. Compute the possible variations of $r_{i, j}$ from values $a_{1}, a_{2}, \cdots, a_{n}$.

3. Compute the rewrite rules from the longest and the most frequent subwords in $S$.

4. Fill matrix $R$ and perform the minimization described above.

5. If the variations of $M$ are greater than those allowed by (8), choose the next possible subwords and repeat. Step 4 . 


\section{$5 \quad$ A CFG and a Regular Transduction}

A ('WC ean be derived from the Dol, computed above very easily. Brielly. every tominal Inlier in every mlo $\phi(t)=s, l \in T^{\prime}, s \in T^{*}$ is replared by a new nonteminal symbol and by alding the related "tominal rules". ( iven $l)=(T, \phi, s)$, let us consider an auxiliary set $N$ and a one-to-one mapping $\mu: T \rightarrow N$

\section{Definition 1.}

$$
G_{D}=(T, N, \mu(s),\{\mu(t) \rightarrow \mu(\phi(t)), l \in T\} \cup\{\mu(l) \rightarrow t, t \in T\})
$$

Moreover, one has to add significant rules for changing these "perfect," words into the real ones. Practical results showed that it is unwise to add these rules diredly to the grammar: the diversity of rurves accepted by a syntactic parser gets too wide. Thus, we decided to model the difference between the context-free language and the real samples by a transduction $\gamma_{S}$, that is a subset $T^{-} \times T^{+}$ of $T^{\star} \times T^{*}$. Let $1^{-}$(respectively $1^{+}$) denote the cardinal of $T^{-\infty}$ (resp. $T^{+}$). Since the noise is bounded, $\gamma$ is a regular transduction ([2], an ahsolute reference).

Inference of $\gamma_{S}$ is achieved by computing the sequence of distances between the "perfect" words $\phi\left(x_{k}\right), 0<k<p\left(x_{0}\right.$ is unknown) and the real samples $x_{k+1}$. The algorithm for computing $d_{L}\left(\tilde{x}_{k+1}, x_{k}\right)$ yields the trace of the distance, that is, the minimum set of basic operations used to transform any of the two words into the other one. Such a trace defines a set of local transductions $\Gamma(k)=$ $\left\{\gamma_{k}^{1}, \gamma_{k}^{2}, \cdots, \gamma_{k}^{p_{k}}\right\}$ for every $k$; let $\Gamma_{s}$ defines the union of local transductions overall the set $S$ of words: $\Gamma_{S}=\bigcup_{0<k<p} \Gamma(k)$.

The (finite) transduction $\gamma_{S}$ is the union for every $k$ of the local transduction $\gamma_{k}=T_{k}^{-} \times T_{k}^{+}$minimizing $\sum_{1<k<p} l_{k}^{-}$and $\sum_{1<k<p} l_{k}^{+}$simultaneously.

\section{Concluding Remarks}

Syntactic recognition of fractal patterns turns out to be a new promising field of research and applications. Promising, first because it shares a conmon background with usual syntactic pattern recognition: one has to dig into formal languages theory in order to find a strong theoretical framework. In fact, the difference lies only in the nature of the model which is assumed to be fractal. But this point is actually essential, for syntactic models can encode this fractality explicitly. Secondly, this fractality is the key to establishing a fruitful connection between both theories of formal languages and dynamical systems. Fascinating problems such as the modification of geometrical generative power of grammars as a function of the mapping appear at the interface of this connection.

Syntactic techniques exhibit real advantages over numerical and statistical techniques (despite [16], a very pessimistic paper !) in the fractal case. Fractality, considered as a recursion scheme, is naturally encoded by means of CFGs. High-level fractal information is processed at the same level as the disparate information. 


\section{References}

1. Aldallah, A.N., Berstel, J.: Tétraarbres engendrés par des automates finis, LITP, Universiló Paris 6, Pance (1980)

2. Autchet, J.-M., Bonsson, L.: 'Tmustuctions ratiomolles, Masson (1988)

3. Barmsley, M.: liactals everywhere, Acakemic P'ress (1088)

4. Beanzamy, B.: How the roots of a polynomial vary with its coefficients : A local quantitative result, Bull. Canadion de Mathómatiques (1998)

5. Blanc-Talon, J.: How to fill the gap betwen Fractals and Stochastic Context-Frce Grammars?, J. Complexity International (1096)

6. Blanc-Talon, J.: MultiFractal Grammars for more Complexity, NOLTA'05. "NonLinear Theory and its $\Lambda$ pplications" (1905)

7. Blanc-Talon, J.: Generation and Recognition of Fractal sets by using a synlactic approach, J. Complexity International (1994)

8. Dekking, F.M.: Recurrent sets, J. Advances in Mathematics, 78-104, 44 (1982)

0. Fernau, H.: IFS and colles, 7th Int. Meeting of Young Computer Scientists (1992)

10. Bordilm, H.: On the degree of nondelerminism of grammars, 7th Int. Meeding of Young Contputer Scientists (1992)

11. Kravis, S.P., Veldkamp, P., Horowilz, H.(B.: Rendering IFS Rncoded 31) Ohjects on a MasPar MP-1, Tech. Report 22, CSIRO-DIT, Cauberra, Australia, (1092)

12. Massopust, P.R.: Fractal Functions, Fractal Surfaces, and Wavelets, Academic Press (1994)

13. Nolle, M.: Comparison of different methods for generating fractals, 7 th Int. Meeting of Young Computer Scientists (1992),

14. Rozenberg, G., Salomaa, A.: The Mathematical Theory of L-systems, Academic Press (1970)

15. Stewart, G.W., Sun, J.-G.: Matrix Perturbation Theory, Academic Press (1990)

16. Tanaka, E.: Theoretical Aspects of Syntactic Pattern Recognition, J. Pattern Recognition, 7 (1994) 